\title{
Management of Post Endodontic Retreatment Pain With Low Level Laser Therapy
}

\author{
Mohammad Asnaashari ${ }^{1}$, Hengameh Ashraf ${ }^{2}$, Amir Houshang Daghayeghi ${ }^{1}$, Seyed Masoud Mojahedi ${ }^{3}$, Saranaz \\ Azari-Marhabi* ${ }^{*}$ \\ ${ }^{1}$ Laser Application in Medical Sciences Research Center, Shahid Beheshti University of Medical Sciences, Tehran, Iran \\ ${ }^{2}$ Department of Endodontics, School of Dentistry, Shahid Beheshti University of Medical Sciences, Tehran, Iran \\ ${ }^{3}$ Department of Laser, School of Dentistry, Shahid Beheshti University of Medical Sciences, Tehran, Iran \\ ${ }^{4}$ Department of Oral Medicine, School of Dentistry, Shahid Beheshti University of Medical Sciences, Tehran, Iran
}

\author{
*Correspondence to \\ Saranaz Azari-Marhabi, DDS; \\ Department of Oral Medicine \\ School of Dentistry, Shahid \\ Beheshti University of Medical \\ Sciences, Tehran, Iran. \\ Tel:+98-2122413897; \\ Fax:+98-2122403075 \\ Email: \\ saranazazari@yahoo.com
}

Published online 27 June 2017

\begin{abstract}
Introduction: Pharmacotherapy with analgesics and non-steroidal anti-inflammatory drugs has been traditionally used to relief post-operative pain of endodontic treatments. However, due to the side effects reported for these drugs, some efforts have been made to decrease the post-operative pain of the endodontic treatments through laser irradiation. The present study aimed to evaluate the effects of low level laser therapy (LLLT) on the reduction of pain after root canal retreatment.

Methods: In this clinical trial, 61 patients requiring endodontic retreatments in posterior teeth were selected. A single visit endodontic retreatment was performed. After biomechanical preparation, low level laser was irradiated to the buccal and lingual mucosa overlying the apices of the target tooth in the experimental group. In the control group patients received placebo laser to eliminate the probable psychological effects of laser. Laser irradiation was done with a single dose of $808 \mathrm{~nm}$ wavelength (Whitening Lase II- Laser DMC, Samsung, Korea) with $100 \mathrm{~mW}$ power, and dose of 70 $\mathrm{J} / \mathrm{cm}^{2}$ for 80 seconds. Pain severity was recorded before, immediately after and 4, 8, 12, 24 and 48 hours after the treatment by visual analogue scale (VAS). The pain scores were statistically analyzed by chi-square test between 2 groups. The effects of different variables on the post-operative pain experience were also studied by means of Logistic regression.

Results: Pain scores decreased significantly through time until 48 hours after treatment. No significant differences were observed between the 2 modalities regarding pain scores at any time. According to regression analysis, pain severity scores were lower in the laser-irradiated specimens than control groups $(\mathrm{OR}=5.69)$; however, this difference was not statistically significant. Consumption of analgesics after the treatment had significant effect in decreasing post-operative pain experience $(\mathrm{OR}=56)$ while factors of age, gender, laser irradiation, pre-treatment pain scores and education level did not.

Conclusion: Low level laser irradiation had limited effects to decrease pain associated with the endodontic retreatments in the first and second molars; however, more studies are required to assess the effects of different parameters of low level laser in this regard.

Keywords: Endodontic retreatments; Low level laser; Pain; Visual analogue scale.
\end{abstract}

\section{Introduction}

Pain after endodontic treatments is a common side effect with significant effect on quality of life of patients. Various degrees of pain have been reported by more than $40 \%$ of endodontic patients after treatment. Generally postoperative endodontic pain is induced by the inflammatory mediators produced via mechanical, chemical, and microbial injuries to the pulp or periradicular tissues, which are induced or exacerbated during root canal treatment and re-treatment. These mediators activate sensitive nociceptors and lead to central and peripheral hyperalgesia mechanisms. ${ }^{1-4}$

In previous studies, the incidence of severe pain after treatment (flare-up) in retreatment cases has been reported to be significantly higher than for primary root canal treatment, emphasizing the need for a proper adapted method to prevent pain complication in retreatment cases. ${ }^{5-8}$

To reduce the intensity of pain, the use of different modalities, such as intra canal therapy, analgesics or nonsteroidal anti-inflammatory drugs (NSAIDs) have been recommended. The therapeutic properties of lasers, such as tissue repair, regulation of inflammatory processes, and analgesia in acute and chronic pain as well as improvement of local microcirculation have been postulated by several authors. ${ }^{7,8}$

Please cite this article as follows: Asnaashari M, Ashraf H, Daghayeghi AH, Mojahedi SM, Azari-Marhabi S. Management of post endodontic retreatment pain with low level laser therapy. J Lasers Med Sci. 2017;8(3):128-131. doi:10.15171/jlms.2017.23. 
The anti-inflammatory and regenerative effects of lowlevel laser therapy (LLLT) have been well established in clinical dentistry, and due to the relatively large side effects of NSAIDs and limitations of use for some of them, safer treatment modalities such as low level laser radiation have been taken into consideration by some researchers. Despite initial promising results, there is not sufficient evidence on the grounds that lasers could be effective in reducing pain after endodontic retreatment or not?

\section{Methods}

This study was a randomized clinical trial. Research data were obtained using questionnaires, interviews, physical examination and classification of pain intensity visual analogue scale (VAS) scores in groups of no pain, mild pain, moderate pain, severe pain and severe pain. In this clinical trial, 61 patients requiring endodontic retreatments in posterior teeth were selected. A single visit endodontic retreatment was performed. After biomechanical preparation, low level laser was irradiated to the buccal and lingual mucosa overlying the apices of the target tooth in the experimental group. In the control group patients received placebo laser to eliminate the probable psychological effects of laser. Laser irradiation was done with a single dose of $808 \mathrm{~nm}$ wavelength (Whitening Lase II- Laser DMC, Samsung, Korea) with $100 \mathrm{~mW}$ power, fiber diameter $600 \mu \mathrm{m}$ and dose of $70 \mathrm{~J} /$ $\mathrm{cm}^{2}$ for 80 seconds. Pain severity was recorded before the treatment, immediately after the treatment and 4, 8, 12, 24 and 48 hours after the treatment by VAS. The pain scores were statistically analyzed by chi-square test between 2 groups. The effects of different variables on the postoperative pain experience were also studied by means of Logistic regression.

\section{Results}

A total of 61 patients, 41 patients $(67.2 \%)$ in the laser group and 20 patients $(32.8 \%)$ in the control group were evaluated. Patients were age matched in both groups. At the beginning of treatment in the laser group, 30 patients (49.2\%) had no pain and 11 patients (18\%) reported pain. In the control group, from the 20 patients studied, 14 patients $(23 \%)$ had no pain and $6(9 \%)$ had pain.

The chi-square test revealed that there was no significant difference among the groups for pain at baseline $(P<0.07)$. In the laser group after treatment, 20 patients $(32.8 \%)$ had no pain, 20 patients $(32.8 \%)$ had mild pain, and $1(1.6 \%)$ had moderate pain. In the control group, 12 patients $(37.5 \%)$ had no pain, and $8(13.1 \%)$ had mild pain. Chi-square test revealed that there was no significant difference in term of pain frequency after treatment in both groups.

Table 2 demonstrates pain scores 4 hours after treatment in laser and control groups. Chi-square test revealed that there was no significant difference in terms of frequency and intensity of pain at 4 hours after treatment in both groups $(P<0.84)$.

Table 3 demonstrates pain scores 8 hours after treatment in laser and control groups. Chi-square test reveals that there was no significant difference in term of frequency and pain intensity 8 hours after treatment in both groups $(P<0.44)$.

Table 4 demonstrates pain scores 12 hours after treatment in laser and control groups. Chi-square test reveals that there was no significant difference in term of in term of frequency and pain intensity 12 hours after treatment in both groups $(P<0.62)$.

Table 5 demonstrates pain scores 24 hours after treatment in laser and control groups. Chi-square test revealed that there was no significant difference in term of in term of frequency and pain intensity 24 hours after treatment in both groups $(P<0.69)$.

Table 6 demonstrates pain scores 48 hours after treatment in laser and control groups. Chi-square test reveals that there was no significant difference in term of in term of frequency and pain intensity 48 hours after treatment in

Table 1. Pain Reported After Treatment in Laser and Control Groups

\begin{tabular}{lcc}
\hline \multirow{2}{*}{ Pain } & Group & \\
\cline { 2 - 3 } & Laser, No. $(\mathbf{\%})$ & Control, No. $(\%)$ \\
\hline No pain & $20(62.5)$ & $12(37.5)$ \\
Mild pan & $20(71.4)$ & $8(28.6)$ \\
Moderate pain & $41(100)$ & $0(0)$ \\
Total & $41(67.2)$ & $20(32.8)$ \\
\hline
\end{tabular}

Table 2. Pain Reported 4 hours After Treatment in Laser and Control Groups

\begin{tabular}{lcc}
\hline \multirow{2}{*}{ Pain } & Group & \\
\cline { 2 - 3 } & Laser, No. $(\%)$ & Control, No. $(\%)$ \\
\hline No pain & $22(36.1)$ & $12(19.7)$ \\
Mild pan & $13(21.3)$ & $5(8.2)$ \\
Moderate pain & $3(4.9)$ & $1(1.6)$ \\
Severe pain & $2(3.3)$ & $2(3.3)$ \\
Very severe pain & $1(1.6)$ & $0(0.0)$ \\
\hline
\end{tabular}

Table 3. Pain Reported 8 hours After Treatment in Laser and Control Groups

\begin{tabular}{lcc}
\hline \multirow{2}{*}{ Pain } & \multicolumn{2}{c}{ Group } \\
\cline { 2 - 3 } & Laser, No. (\%) & Control, No. (\%) \\
\hline No pain & $22(36.1)$ & $13(21.3)$ \\
Mild pan & $14(23.0)$ & $4(6.6)$ \\
Moderate pain & $2(3.3)$ & $1(1.6)$ \\
Severe pain & $1(1.6)$ & $2(3.3)$ \\
Very severe pain & $2(3.3)$ & $0(0.0)$ \\
\hline
\end{tabular}

Table 4. Pain Reported 12 hours After Treatment in Laser and Control Groups

\begin{tabular}{lcc}
\hline \multirow{2}{*}{ Pain } & Group & \\
\cline { 2 - 3 } & Laser, No. $(\%)$ & Control, No. $(\%)$ \\
\hline No pain & $22(36.1)$ & $14(23.0)$ \\
Mild pan & $13(21.3)$ & $3(4.9)$ \\
Moderate pain & $2(3.3)$ & $1(1.6)$ \\
Severe pain & $3(4.9)$ & $2(3.3)$ \\
Very severe pain & $1(1.6)$ & $0(0.0)$ \\
\hline
\end{tabular}


Table 5. Pain Reported 24 hours After Treatment in Laser and Control Groups

\begin{tabular}{lcc}
\hline \multirow{2}{*}{ Pain } & Group & \\
\cline { 2 - 3 } & Laser, No. $(\%)$ & Control, No. (\%) \\
\hline No pain & $27(44.3)$ & $15(24.6)$ \\
Mild pan & $9(14.8)$ & $2(3.3)$ \\
Moderate pain & $2(3.3)$ & $1(1.6)$ \\
Severe pain & $2(3.3)$ & $2(3.3)$ \\
Very severe pain & $1(1.6)$ & $0(0.0)$ \\
\hline
\end{tabular}

Table 6. Pain Reported 48 hours After Treatment in Laser and Control Groups

\begin{tabular}{lcc}
\hline \multirow{2}{*}{ Pain } & Group & \\
\cline { 2 - 3 } & Laser, No. $(\mathbf{\%})$ & Control, No. $\mathbf{( \% )}$ \\
\hline No pain & $28(45.9)$ & $16(26.2)$ \\
Mild pan & $9(14.8)$ & $3(4.9)$ \\
Moderate pain & $2(3.3)$ & $1(1.6)$ \\
Severe pain & $2(3.3)$ & $0(0.0)$ \\
Very severe pain & $0(0.0)$ & $0(0.0)$ \\
\hline
\end{tabular}

both groups $(P<067)$.

In the laser group before treatment, 31 patients $(50.8 \%)$ did not use pain killer medication, and 10 patients (16.4\%) used these drugs. In the control group, 15 patients (24.6\%) did not use pain killer medication and only 5 patients $(8.2 \%)$ had used these drugs. No significant difference in analgesic consumption was observed in both groups before treatment $(P=0.96)$.

In the laser group after treatment, 37 patients (60.7\%) did not use painkiller medication, and 4 patients (6.6\%) had used these drugs. In the control group, 16 patients $(26.2 \%)$ did not use painkiller medication, and 4 patients (6.6\%) had used these drugs. No significant difference in analgesic consumption was observed in both groups after treatment $(P=0.26)$.

In the laser group, 20 subjects (32.8) had pain in maxilla and 21 patients $(34.4 \%)$ had pain in mandible. In the control group, 10 patients $(16.4 \%)$ had pain in the maxilla and 10 patients $(16.4 \%)$ had pain in the mandible. No Significant differences were observed in the frequency of pain in the maxillary and mandibular position in patients undergoing laser therapy and the control group $(P=0.93)$. In the laser group 17 patients $(27.9 \%)$ had pain in the right quadrant and 24 patients (39.3\%) had pain in the left quadrant. In the control group, the location of pain in 11 patients (18\%) was on the right side and in 9 patients $(14.8 \%)$ on the left quadrant. No Significant differences were observed in the frequency of pain position in laser and control groups $(P=0.32)$.

In the laser group 34 patients (55.7\%) reported tenderness and in 7 patients $(11.5 \%)$ teeth were not tender. In the control group, 11 teeth (18\%) were tender and $9(14.8 \%)$ were not tender. There was significant difference in tooth tenderness in patients in laser and control groups $(P<0.02)$

In the laser group 20 patients $(32.8 \%)$ had periapical radiolucency and in 21 patients $(34.4 \%)$ no radiolucency was observed. In the control group, 10 teeth $(16.4 \%)$ had no radiolucency and 10 teeth (16.4\%) had radiolucency. No significant difference in periapical radiolucency was observed in laser and control groups $(P<0.98)$.

Friedman test showed that over time pain scores were significantly reduced in both laser $(P<0.05)$ and control $(P<0.004)$ groups. In order to investigate the relation between each of the variables and degree of pain reduction, the ordinal logistic regression was used and the results showed that the only factor associated with significant pain reduction was using medications in both laser and control groups. Other variables did not show a significant relationship with pain scores. According to regression analysis, pain severity scores were lower in the laser-irradiated specimens than control groups (odds ratio $[\mathrm{OR}]=5.69$ ); however, this difference was not statistically significant. Consumption of analgesics after treatment had significant effect to decrease postoperative pain experience $(\mathrm{OR}=56)$ while factors such as age, gender, laser irradiation, pre-treatment pain scores and education level did not.

\section{Discussion}

Pain has been reported after root canal treatment and retreatments in several studies, which has been estimated to be $1.4 \%$ to $16 \%{ }^{9}$ Previous research, have shown that factors such as age, sex, dental pulp status, allergies and pain before treatment have a primary role in causing these pains. ${ }^{10}$

Although the non-steroidal anti-inflammatory drugs for pain associated with endodontic therapy have been used, these medications have relatively large side effects in some patients. Therefore, the use of laser for pain associated with endodontic therapy aimed at eliminating these complications. However, more research should be done on the effects of laser after endodontic therapy to reduce pain. Mechanisms for reducing pain following laser radiation has been related to lowering levels of prostaglandin E2, prostaglandin-endoperoxide synthase 2, interleukin 1-beta, tumor necrosis factor-alpha, the cellular influx of neutrophil granulocytes, oxidative stress, edema, and bleeding. ${ }^{11}$

According to the results of the present study, in both groups (low level laser radiation and control groups) the degree of pain was significantly reduced up to 48 hours after treatment while no significant differences were observed between the 2 groups in terms of pain intensity (at no time). On the other hand, according to the results of the regression analysis, pain reduction in radiated samples was 5 times higher than the control group $(\mathrm{OR}=$ 5.69), but this difference was not statistically significant. Accordingly; low-level laser radiation had limited effects in reducing pain following root canal re-treatment in posterior teeth. Based on researchers' information, until now, no research on the effects of laser radiation on the amount of pain associated with root canal re-treatment has been published, although some studies have been conducted on the analgesic effects of laser radiation. Based 
on the results of the present study, none of the variables, such as age, sex, pain before treatment influenced the intensity of pain after root canal re-treatment.

Lim et $\mathrm{al}^{10}$ reported reduction of pain following laser irradiation in patients receiving orthodontic treatments. Also Kreisler et al $^{12}$ stated that pain scores were reduced in both laser and control groups after endodontic surgery. These findings are consistent with the results of present study.

Pain after root canal treatment depends on the extent of the periradicular tissue injury, severity and intensity of the inflammatory response. These factors are interrelated and directly interdependent, followed by acute inflammation such as periodontitis, pre radicular acute or chronic abscess, while subsequent processes occurred within the channel. ${ }^{13}$

It has been stated that pain before endodontic treatment affects pain intensity after treatment, although the results of this study showed no significant differences in pain intensity and frequency in both therapy and control groups. $^{11}$

In both laser and control groups; Pain severity was significantly reduced up to 48 hours after treatment and no significant difference in pain intensity between the two groups was found at any time of the study.

According to regression analysis, the degree of pain reduction in irradiated samples was higher than the control group $(\mathrm{OR}=5.69)$, but this difference was not statistically significant.

Among the variables studied such as age, gender, laser irradiation, and pain before the intervention, using analgesics was the only factor which significantly affected the post-operative pain.

\section{Conclusion}

Low level laser irradiation had limited effects in decreasing pain associated with endodontic retreatments in the first and second molars; however, more studies are required to assess the effects of different parameters of low level laser in this regard.

\section{Conflict of Interests}

All the authors certify that they have no involvement in any organization or entity with any financial or nonfinancial interest in the subject matter or materials discussed in this manuscript.

\section{Ethical Considerations}

This clinical trial was done according to the Declaration of Helsinki. Also the protocol was approved by the ethics committee of Shahid Beheshti University of Medical Sciences (SBMU.REC.1392.681) and it was registered in the US National institute of clinical trials (identifier: NCT03023761).

\section{References}

1. Maiman TH. Stimulated optical radiation in ruby. Nature. 1960;187:493-494

2. Genet J, Wesse L, Link P, Thoden S. The incidence of preoperative and postoperative pain in endodontic therapy. Int Endod J. 1986;20:53-64.

3. Hagreaves KM, Cohen S. Pathways of the Pulp. 10th ed. Missouri: Mosby; 2011.

4. Pasqualini D, Mollo L, Scotti N, et al. Post-operative pain after manual and mechanical glide path: a randomized clinical trial. J Endod. 2012;38(1):32-36. doi:10.1016/j. joen.2011.09.017.

5. Taintor JF, Langeland K, Valle GF, Krasny RM. Pain: a poor parameter of evaluation in dentistry. Oral Surg Oral Med Oral Pathol Oral Radiol Endod. 1981;52:299-303.

6. Torabinejad M, Kettering JD, McGraw JC, Cummings RR, Dwyer TG, Tobias TS. Factors associated with endodontic inter-appointment emergencies of teeth with necrotic pulps. J Endod. 1988;14:261-266.

7. Trope M. Flare-up rate of single visit endodontics. Int Endod J. 1991;24:24-26.

8. Wang C, Xu P, Ren L, Dong G, Ye L. Comparison of post-obturation pain experience following one-visit and two-visit root canal treatment on teeth with vital pulps: a randomized controlled trial. Int Endod J. 2010;43(8):692697. doi:10.1111/j.1365-2591.2010.01748.x.

9. Ng YL, Gennon JP, Setchell DJ, Gulabivala K. Prevalence of and factors affecting post-obturation pain in patients undergoing root canal treatments. Int Endod J. 2004;37:381391.

10. Lim HM, Lew KK, Tay DK. A clinical investigation of the efficacy of low level laser therapy in reducing orthodontic postadjustment pain. Am J Orthod Dentofacial Orthop. 1995;108(6):614-622

11. Asnaashari M, Mohebi SH, Paymanpour P. Pain reduction using low level laser irradiation in single-visit endodontic treatment. J Lasers Med Sci. 2011;2(4):139-143.

12. Kreisler MB, Haj HA, Noroozi N, Willershausen B, d'Hoedt B. Efficacy of low level laser therapy in reducing postoperative pain after endodontic surgery- a randomized double blind clinical study. Int J Oral Maxilofacial Surg. 2004;33(1):38-41.

13. Walton R, Fouad A. Endodontic interappointment flareups: a prospective study of incidence and related factors. $J$ Endod. 1992;18:172-177. 